\title{
Can the Elastic of Surgical Face Masks Stimulate Ear Protrusion in Children?
}

\author{
Bruno Zanotti ${ }^{1}$ - Pier Camillo Parodi ${ }^{2} \cdot$ Michele Riccio $^{3}$ - Francesco De Francesco ${ }^{3}$. \\ Nicola Zingaretti ${ }^{2}$ (i)
}

Received: 3 June 2020/ Accepted: 4 June 2020/Published online: 18 June 2020

(C) Springer Science+Business Media, LLC, part of Springer Nature and International Society of Aesthetic Plastic Surgery 2020

\begin{abstract}
In this period of the Covid-19 pandemic, a protective mask has become a common object of use to contain virus transmission. The imminent need for masks has led many governments to produce them, including surgical masks with elastic loops or masks with side cuts at the ears. Among those on the market, surgical masks with elastic loops are the ones most chosen by parents for their children. These elastics cause constant compression on the skin and, consequently, on the cartilage of the auricle, leading to erythematous and painful lesions of the retroauricular skin when the masks are used for many hours a day. Pre-adolescent children have undeveloped auricular cartilage with less resistance to deformation; prolonged pressure from the elastic loops of the mask at the hollow or, even worse, at the anthelix level can influence the correct growth and angulation of the outer ear. In fact, unlike when using conservative methods for the treatment of protruding ears, this prolonged pressure can increase the cephaloauricular angle of the outer auricle. It is important for the authorities supplying the masks to be aware of this potential risk and for alternative solutions to be found while maintaining the possibility of legitimate prevention of the potential spread of the virus.
\end{abstract}

Nicola Zingaretti

zingarettin@gmail.com

1 Department of Neurosurgery, Ospedale Carlo Poma, Mantova, Italy

2 Clinic of Plastic and Reconstructive Surgery, Department of Medical Area (DAME), Academic Hospital of Udine, University of Udine, Udine, Italy

3 Department of Plastic Reconstructive Surgery and Hand Surgery, Azienda Ospedaliero Universitaria Ospedali Riuniti, Ancona, Italy
Level of Evidence $V$ This journal requires that authors assign a level of evidence to each article. For a full description of these evidence-based medicine ratings, please refer to the table of contents or the online instructions to authors www.springer.com/00266.

Keyword Ear protrusion - External ear - Surgical face mask $\cdot$ Infant $\cdot$ Covid-19

Dear Sir,

We read with interest the article by Ors [1] on prominent ear deformities and its recurrence rate. This article gives us the opportunity to better understand the effectiveness of nonsurgical options for the treatment of ear anomalies in young patients (5-14 years).

The current COVID-19 pandemic has imposed the worldwide use of masks in addition to social distancing. The use of masks applies to everyone, even children, certainly if over the age of 5-6 years.

The masks distributed to the population by government bodies are of various types, both as regards to their shape and the systems for fastening them to the head, but, basically, they are divided into 4 large categories: masks with elastic (ear loops), strips of fabric with lateral slits (side cuts at the ears), with tapes, single cervical band.

Small children are rarely made to wear the masks with tapes, due to the difficulty in positioning and tying the tapes.

The "single band" masks that wrap around the neck have the drawback of sliding downwards and, therefore, not keeping the nose covered; furthermore, if used during the summer season, they tend to produce a humid 
microenvironment that favours the development of dermatitis and eczema.

Consequently, the masks most used for children are the first two types (Fig. 1a and b).

Many adults (health care workers and others) complain about discomfort associated with ear loops, due to the continuous pressure of the elastic behind the ear. Several methods have been proposed to overcome this problem: from the use of hairpins to hang the elastic bands from the forehead to the use of various types of bands that pass behind the back of the neck and to which the loops of the mask are attached. However, these methods used by adults are not used by children, resulting in constant pressure of the elastic on the skin of the posterior portion of the auricle.

All bodies subject to the action of a force undergo deformation, which depends not only on the intensity of the force applied, but also on the nature of the body itself. In general, deformations can be of two types: elastic, which disappear when the force is no longer applied, and nonelastic, which remain even after applying the force. Cartilage has memory thanks to the presence of elastic fibres; although this allows it to return to its initial condition when deformed, if the stimulus persists, it can lead to permanent changes in its conformation.

This principle has been used for many years for the conservative correction of protruding ears $[1,2]$. Obviously, in order to use these non-surgical correction techniques (e.g. bands, patches, ear splint therapy) with benefit, the development of the various components of the ear must not be completed. This can happen if applied in children who are less than 8-10 years old, because after that age the cranial volume, with its appendages, almost completely reaches total growth [3]. The literature reports important successes in the treatment of protruding ears with conservative methods, with a success rate that shows a decline from $91 \%$ in infants to $33 \%$ in 9-year-old children $[1,2,4]$.

The main advantage of this treatment is that it keeps the splint in position for a long time, so that it can exert constant pressure on the growing cartilage, changing it into the desired shape.

The success of these conservative methods confirms that a constant pressure maintained for a long time on the cartilage of the ear of growing children can change the shape and increase the cephaloauricular angle of the outer auricle.

Griffin et al. have demonstrated that the cartilage of the auricle has a homogeneous structure, although the concha has a greater resistance to deformation than the helix [5]. This resistance is comparable to that of nasal cartilage (about 200 times lower than the resistance of Medpor prostheses used for ear reconstruction). The concha had a greater rate of loading than the antihelix when taking into consideration the anatomical structure of the cartilages. One reason for this difference may be that the concha is a curved structure, which can support compressive loads [5]. Therefore, if a constant elastic force were applied at the level of the concha, it would be less harmful than if applied at the antihelix level.

It is well known that the pressure of the elastic on the skin of the posterior concha of the auricle for a long time causes pain and erythema, due to the continuous rubbing of the elastic on the skin in the same position. This has led some users to apply silicone rear ear supports that decrease decubitus, but increase the distance of the auricle from the
Fig. 1 The masks most used for children. a Surgical mask with ear loops. b Mask with side cuts at the ears
A
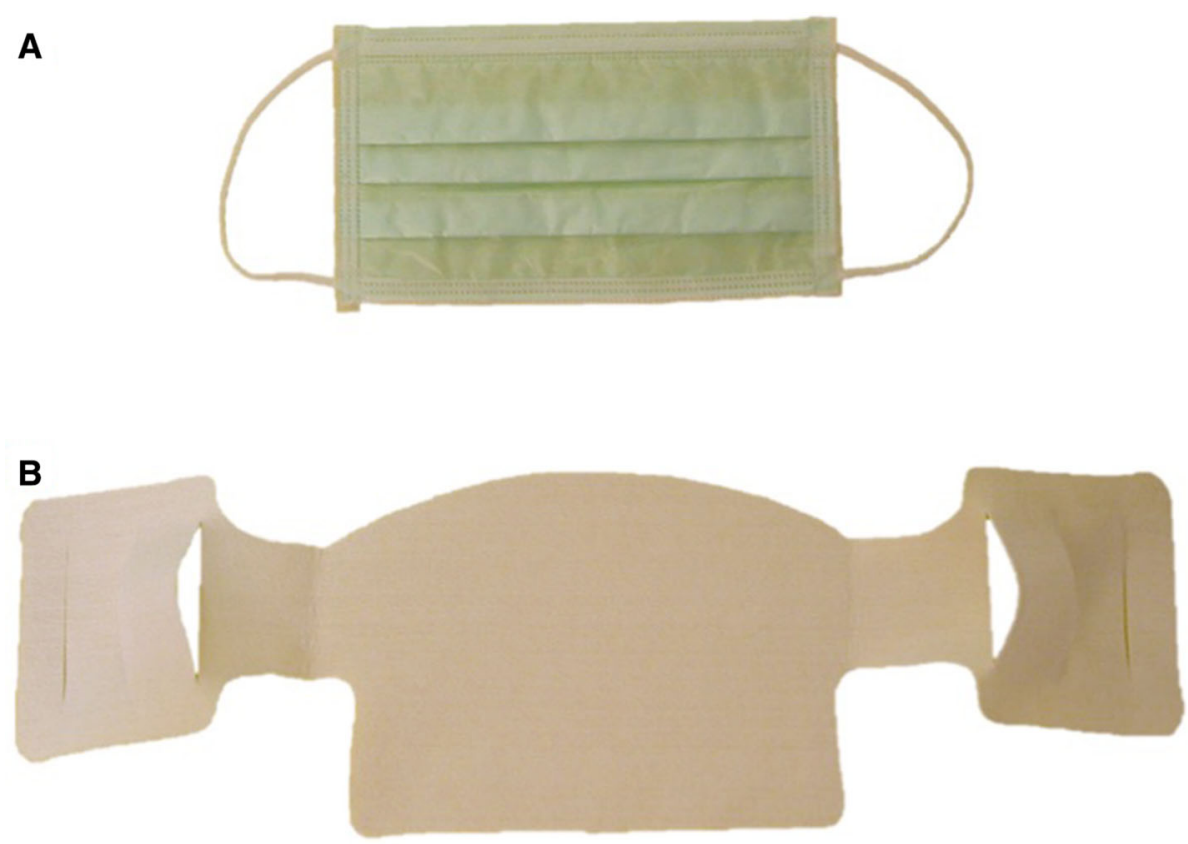

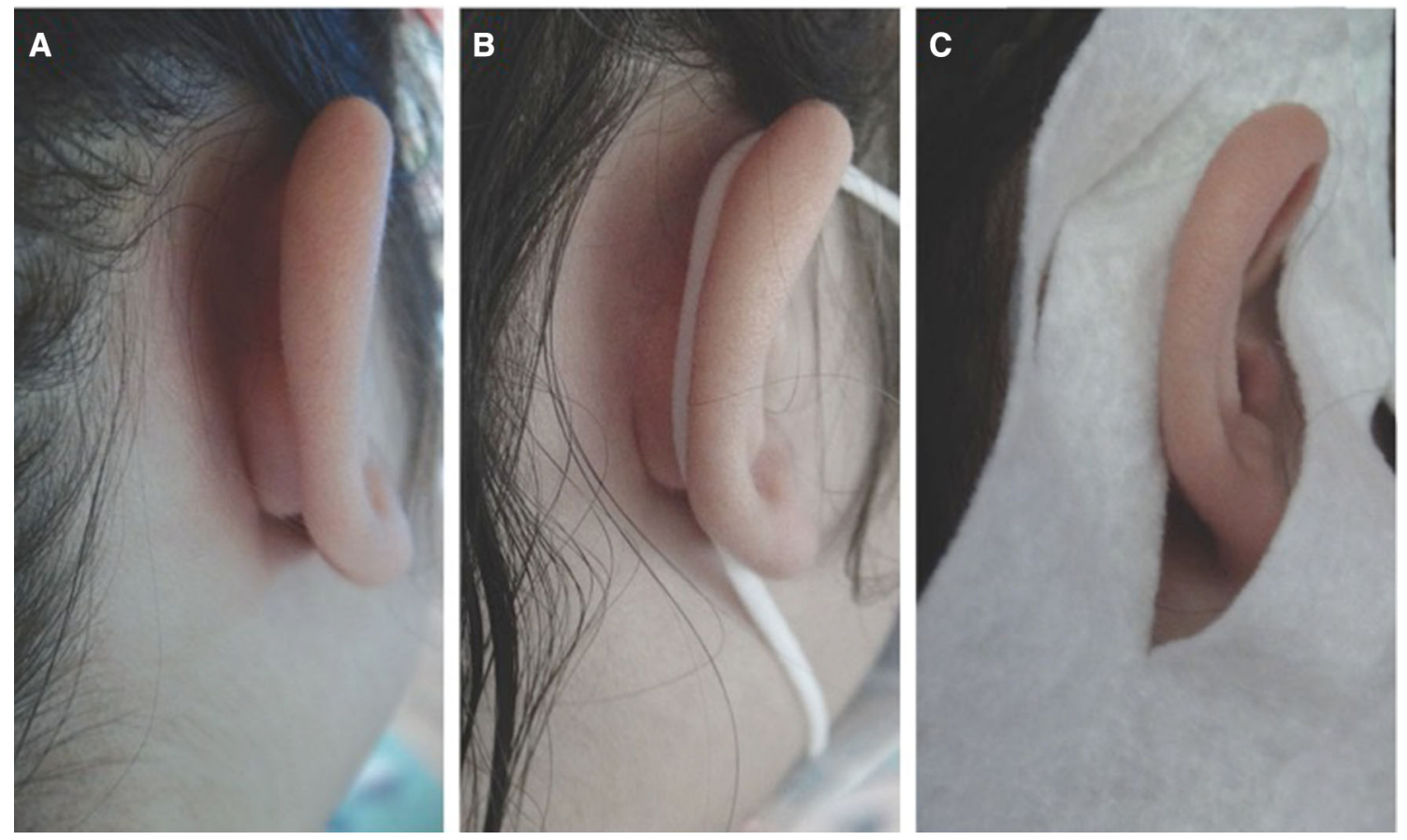

Fig. 2 a Modification of the cephaloauricular angle following the placement of a surgical mask with ear loops (b) and band mask with side slits (c) in a 6-year-old girl

mastoid region. Others, on the other hand, prefer to change the position of the elastic by moving it more towards the antihelix than the mastoid region, thus applying an elastic force in an area where the cartilage has less resistance, with a consequent increase in the cephaloauricular angle of the outer auricle.

Therefore, the use of surgical masks with ear loops in growing children for many hours a day not only leads to intolerance and decubitus of the retroauricular skin (as for adults), but can also influence the correct growth and angulation of the outer ear with the consequent increase in the incidence of protrusion of the outer auricle (Fig. 2a and b).

One alternative to the surgical mask is the earmuffs mask (in which the ears are completely wrapped, held in place by an elastic band at the back of the ear) or the band mask with lateral slits at the ears: also these models, due to their conformation, tend to create a constant pressure on the antihelix, increasing the auriculocephalic angle (Fig. 2c).

Many scientific societies of paediatrics and pedagogy have raised doubts about the risks that this situation of forced constriction by Covid-19 can cause on the normal psychophysical development of children; however, it has never been pointed out how the use of surgical masks with ear loops can be harmful to the correct development of the auricle by permanently modifying the cartilage of the concha in growing children.

We are confident that highlighting this potential complication to those involved in the procurement of masks will stimulate the search for alternative solutions, while maintaining the possibility of legitimate prevention of the potential spread of the virus.

\section{Compliance with Ethical Standards}

Conflict of interest The authors declare that they have no conflicts of interest to disclose.

Human and Animal Rights This article does not contain any studies with human participants or animals performed by any of the authors.

Informed Consent For this type of study informed consent is not required.

\section{References}

1. Ors S (2020) Comparison of prominent ear recurrence in different age groups. Aesthet Plast Surg. https://doi.org/10.1007/s00266020-01757-4(In press)

2. Woo JE, Park Y-H, Park EJ, Park KY, Kim SH, Yim S-Y (2017) Effectiveness of ear splint therapy for ear deformities. Ann Rehabil Med 41:138-147 
3. Zanotti B, Zingaretti N, Verlicchi A, Robiony M, Alfieri A, Parodi PC (2016) Cranioplasty: review of materials. J Craniofac Surg 27:2061-2672

4. Yotsuyanagi $T$ (2004) Nonsurgical correction of congenital auricular deformities in children older than early neonates. Follow up. Plast Reconstr Surg 114:190-191

5. Griffin MF, Premakumar Y, Seifalian AM, Szarko M, Butler PEM (2016) Biomechanical characterisation of the human auricula cartilages; Implication for tissue engineering. Ann Biomed Eng 44:3460-3467

Publisher's Note Springer Nature remains neutral with regard to jurisdictional claims in published maps and institutional affiliations. 